OPEN ACCESS

Edited by:

Cheryl M. Glazebrook,

University of Manitoba, Canada

Reviewed by:

Xiaoyi $\mathrm{Hu}$,

Beijing Normal University, China

Przemyslaw Tomalski,

University of Warsaw, Poland

*Correspondence:

Leonardo Zapata-Fonseca zapatafonseca.leonardo@gmail.com Bert Timmermans bert.timmermans@abdn.ac.uk

Specialty section This article was submitted to

Developmental Psychology, a section of the journal

Frontiers in Psychology

Received: 27 August 2018 Accepted: 21 December 2018

Published: 11 January 2019

Citation:

Zapata-Fonseca L, Dotov D, Fossion R, Froese T, Schilbach L, Vogeley K and Timmermans B (2019) Multi-Scale Coordination of Distinctive Movement Patterns During Embodied Interaction Between Adults With

High-Functioning Autism and Neurotypicals.

Front. Psychol. 9:2760. doi: 10.3389/fpsyg.2018.02760

\section{Multi-Scale Coordination of Distinctive Movement Patterns During Embodied Interaction Between Adults With High-Functioning Autism and Neurotypicals}

\author{
Leonardo Zapata-Fonseca1,2*, Dobromir Dotov ${ }^{3}$, Ruben Fossion ${ }^{2,4}$, Tom Froese ${ }^{2,5}$, \\ Leonhard Schilbach ${ }^{6,7}$, Kai Vogeley ${ }^{8,9}$ and Bert Timmermans ${ }^{10 *}$
}

\footnotetext{
${ }^{1}$ Plan of Combined Studies in Medicine (PECEM), Faculty of Medicine, National Autonomous University of Mexico, Mexico City, Mexico, ${ }^{2}$ Center for the Sciences of Complexity (C3), National Autonomous University of Mexico, Mexico City, Mexico, ${ }^{3}$ Research and High Performance Computing, LIVELab, McMaster University, Hamilton, ON, Canada, ${ }^{4}$ Department of Matter Structure, Nuclear Sciences Institute, National Autonomous University of Mexico, Mexico City, Mexico, ${ }^{5}$ Department of Computer Science, Institute of Applied Mathematics and Systems Research, National Autonomous University of Mexico, Mexico City, Mexico, ${ }^{6}$ Independent Max Planck Research Group for Social Neuroscience, Max Planck Institute of Psychiatry, Munich, Germany, ' Department of Psychiatry, Ludwig-Maximilians-Universität München, Munich, Germany, ${ }^{8}$ Department of Psychiatry and Psychotherapy, University Hospital Cologne, Cologne, Germany, ${ }^{9}$ Cognitive Neuroscience (INM-3), Institute of Neuroscience and Medicine, Research Center Jülich, Jülich, Germany, ${ }^{10}$ The School of Psychology, University of Aberdeen, Aberdeen, United Kingdom
}

Autism Spectrum Disorder (ASD) can be understood as a social interaction disorder. This requires researchers to take a "second-person" stance and to use experimental setups based on bidirectional interactions. The present work offers a quantitative description of movement patterns exhibited during computer-mediated real-time sensorimotor interaction in 10 dyads of adult participants, each consisting of one control individual (CTRL) and one individual with high-functioning autism (HFA). We applied timeseries analyses to their movements and found two main results. First, multi-scale coordination between participants was present. Second, despite this dyadic alignment and our previous finding that individuals with HFA can be equally sensitive to the other's presence, individuals' movements differed in style: in contrast to CTRLs, HFA participants appeared less inclined to sustain mutual interaction and instead explored the virtual environment more generally. This finding is consistent with social motivation deficit accounts of ASD, as well as with hypersensitivity-motivated avoidance of overstimulation. Our research demonstrates the utility of time series analyses for the second-person stance and complements previous work focused on non-dynamical and performance-based variables.

Keywords: autism spectrum disorder, time-series analysis, social interaction, movement variability, humancomputer interface, tactile interaction, social motor coordination, multi-scale analysis 


\section{INTRODUCTION}

Autism Spectrum Disorder (ASD) can be understood as a social interaction disorder (American Psychiatric Association and American Psychiatric Association DSM-5 Task Force, 2013). This makes the emerging "second-person approach" to social cognition a more promising framework for studying ASD than classical approaches focusing on mind-reading capacities in isolated, detached and observer-based arrangements (Gallagher, 2004; De Jaegher, 2013; Froese et al., 2013). According to this second-person approach, a variety of embodied, perceptual and interactive capabilities are required for the full competence of understanding others (Froese and Di Paolo, 2011; Schilbach et al., 2013; Froese, 2018), all of which are hypothesized to be compromised in ASD (Gallagher, 2004; Fuchs, 2015; Schilbach, 2016). This approach states that, rather than making sense of others through observation or simulation, which occur in the observer, we need to engage with the other person by means of reciprocal action control (Kunde et al., 2018), thereby not simply "understanding" the other, but rather to jointly engage in a set of learned social skills that give us an immediate attunement to the other.

We therefore investigated the capacity to detect social contingencies as the mutual responsiveness of interaction partners in 10 dyads of adult participants engaging in computermediated embodied social interaction. Each dyad consisted of one control individual (CTRL) and one individual with high-functioning autism (HFA). Participants were asked to distinguish the interaction partner from other non-reactive items by clicking. In previous work, we studied the accuracy of this social contingency detection, finding that individuals with HFA did not differ significantly in their clicking accuracy from controls (Zapata-Fonseca et al., 2018). However, individuals with HFA were notably more conservative in their clicking, and visual inspection of their movements revealed marked differences in style compared to controls. These observations in conjunction with recent work on stochastic patterns of motor variability to objectively characterize the ASD phenotype (Torres et al., 2017) motivated us to present this quantitative description of movement patterns during realtime embodied interactions. Our work aims to provide a quantifiable link between on the one hand individual motor movement markers as potential diagnostic tools for ASD (e.g., Cook et al., 2013), and on the other hand social interaction deficits of ASD, which are currently the two main diagnostic criteria.

\section{MATERIALS AND METHODS}

The time series of embodied interaction were recorded by means of a minimalistic human-computer interface paradigm that has become known as the "Perceptual Crossing Experiment" (PCE), originally developed by Lenay (2008); for a review, see Auvray and Rohde (2012). The real-time sensorimotor features of the PCE have proven to be capable of eliciting meaningful social interactions and forms of mutual alignment that are also found in real-life social interactions (Froese et al., 2014; Froese and Zapata-Fonseca, 2017).

\section{Participants}

Participants within one dyad were matched with respect to sex and age. The 10 HFA participants ( 5 male) were between 29 and 54 years of age $(M=42.32, S D=9.20)$ and were diagnosed and recruited at the Autism Outpatient Clinic at the Department of Psychiatry of the University Hospital of Cologne in Germany (one participant was diagnosed at the Cologne Autism Therapy Centre). The diagnoses were confirmed by clinical interviews according to ICD-10 criteria by two specialized physicians and were supplemented by extensive neuropsychological assessment. The sample included patients with the diagnosis "Asperger syndrome" according to ICD-10 with an at least average FullScale IQ (FSIQ N85, measured using Wechsler Adult Intelligence Scale, WAIS).

\section{Perceptual Crossing Experiment (PCE)}

During the PCE, two participants are mutually interacting as embodied avatars within a one-dimensional circular virtual space. Participants control the movement of their respective avatars using a computer mouse and receive information about a direct contact with objects in the virtual space via on-or-off tactile feedback to the hand. They can encounter three types of objects: (i) the other's avatar; (ii) a moving object that "shadows" the other's avatar; and, (iii) a static object (see Figure 1).

The task is to move around freely and to identify the partner's avatar by clicking the mouse button to report a perceived encounter, but not during encounters with either the shadow or the fixed object. The three types of objects produce identical sensory consequences to the hand when a participant's avatar meets them in the virtual space but provide different affordances (potentials) for interaction. In particular, distinguishing between a static object, the partner's moving avatar, and the shadow of a partner's avatar depends on being sensitive to their particular affordances for interaction; only the other's avatar can be responsive. Each dyad performed three trials of $5 \mathrm{~min}$ each. Participants do not receive feedback about their clicking performance until after the experiment.

\section{Time-Series Analysis of Participants' Movement Patterns}

In the PCE, the computer-mediated participants' behavior permits a detailed recording of how their movements and sensations change over time; see Supplementary Figure 2. Timeseries approaches to embodied social cognition shift the research question toward how rather than what tasks involving social capacities are being solved (Zapata-Fonseca et al., 2016; Romero et al., 2018).

\section{Interpersonal Coordination}

Complexity Matching (CM) accounts for generalized coordination between two multi-scale processes. CM is the fit between the power-laws describing how the variance of each process increases as the scale over which it is measured increases. 


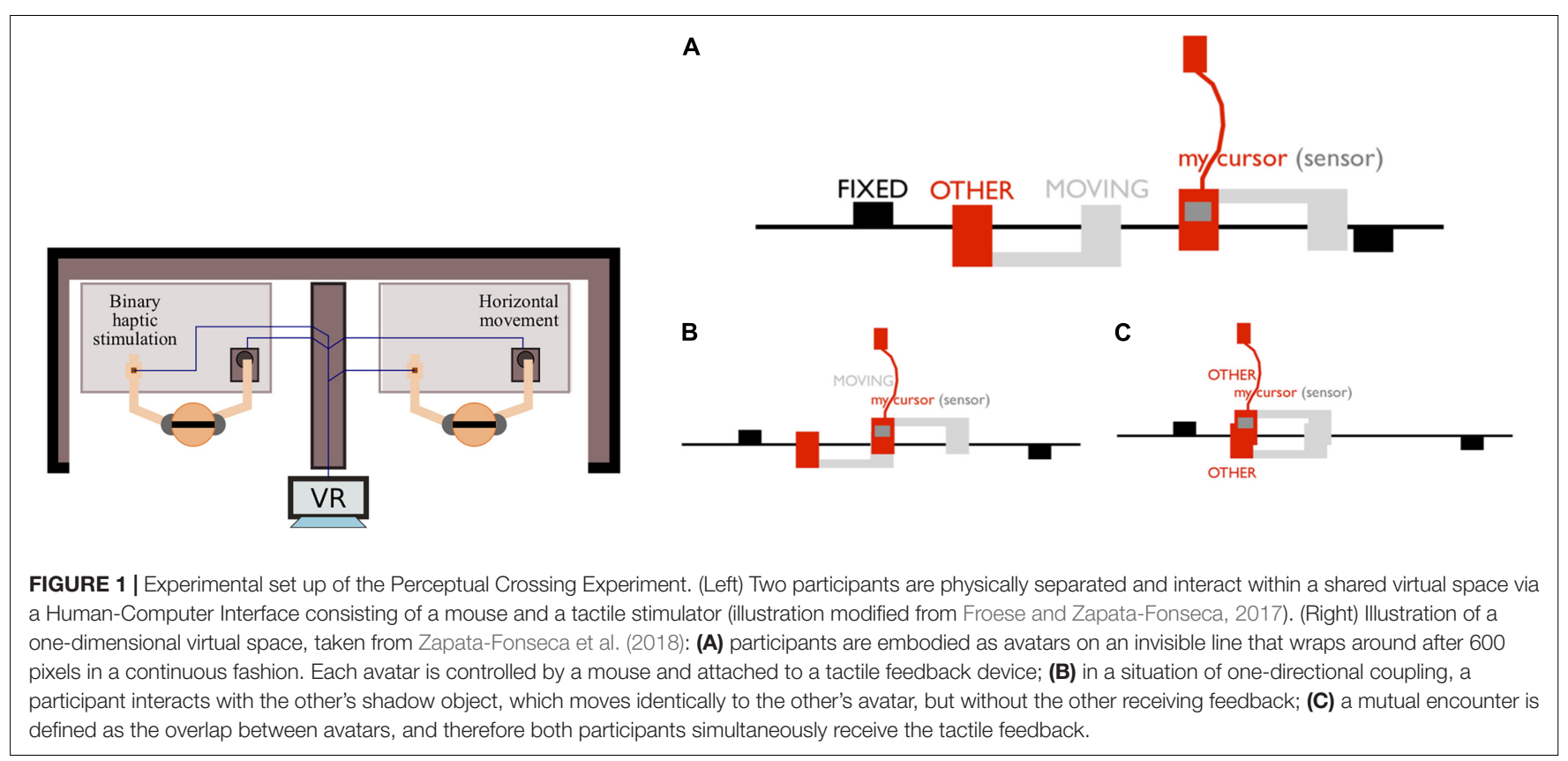

$\mathrm{CM}$ is maximized when information exchange between the processes is maximized (West et al., 2008). For point processes, such as the zero-crossings of avatars' acceleration, the variance is obtained using the Allan Factor (Allan, 1966). This method has been applied to groups of healthy participants (Abney et al., 2014; Zapata-Fonseca et al., 2016). Testing for complexity matching consists of surrogate analysis; see Supplementary Material's Section 1.

\section{Individual Movement Profiles}

To study participants' amount of movement and its variability (Torres et al., 2013; Słowiński et al., 2016), we took the magnitude and standard deviation of the velocities. Velocity was defined as the rate of change in positions (Zapata-Fonseca et al., 2016). From the velocity time series we took the absolute values and analyzed the mean (Speed_Mean) and the standard deviation (Speed_SD), each of which was fitted independently against Group and Trial using hierarchical modeling (Singer and Willett, 2003) in Ime4 (Bates et al., 2015); see Supplementary Material's Section 2.

\section{Multi-Scale Movement Variability}

A more detailed study of the movement variability was made through coarse-grained analysis similar to Fossion et al. (2017). The time series is analyzed at different scales without decomposing it into different non-overlapping components, but instead incrementally filtering out finer scales such that the coarse-grained time series at finer scales will still contain the coarse features and only at the coarser scales the finer details will be filtered out (see Supplementary Figure 3). This allows to quantify how much small-scale and especially large-scale components account for the variability of a signal by computing its variance according to different resolution factors. In the context of the PCE small-scale fluctuations correspond to jittery movements, whereas large-scale components indicate sustained movements in one direction; see Supplementary Material's Section 3.

\section{RESULTS}

\section{Complexity Matching Between Controls and Patients With HFA}

Complexity matching between participants was present as revealed by the surrogate dyads analysis: $\mathrm{D}_{\mathrm{a}, \mathrm{b}}$ Original $(M=12.84$, $S D=3.3)$ was significantly higher than $\mathrm{D}_{\mathrm{a}, \mathrm{b}}$ Surrogate $(M=11.35$, $S D=1.06), t(29)=2.46, p=0.01$ (see Supplementary Figure 1). The difference between similarity indices $\left(D_{a, b}\right.$ Original vs $D_{a, b}$ Surrogate) means that the structure of the movements' variability across time scales was globally coordinated between the real dyads (participants).

\section{Speed Profiles Distinguish Between Controls and Patients With HFA}

For both dependent variables the significant coefficients can be found in Supplementary Table 1. Figure 2A shows the form of the fitted model: whereas numerically the CTRL group appears to have both higher Speed_Mean and Speed_SD than the HFA group, the factor Group did not contribute significantly to a model including Trial and the Trial:Group interaction as predictors, so, overall, the average Speed_Mean and Speed_SD did not differ between groups. Instead, there was a main effect of Trial $(p=0.059)$, for Speed_Mean, showing that mean speed increased over trials, without a significant interaction with Group. For Speed_SD on the other hand, there was no main effect of Trial, but there was an interaction between Trial and Group ( $p=0.049$ ), suggesting that only for CTRL did Speed_SD (variability in movements) increase over trials. 
A
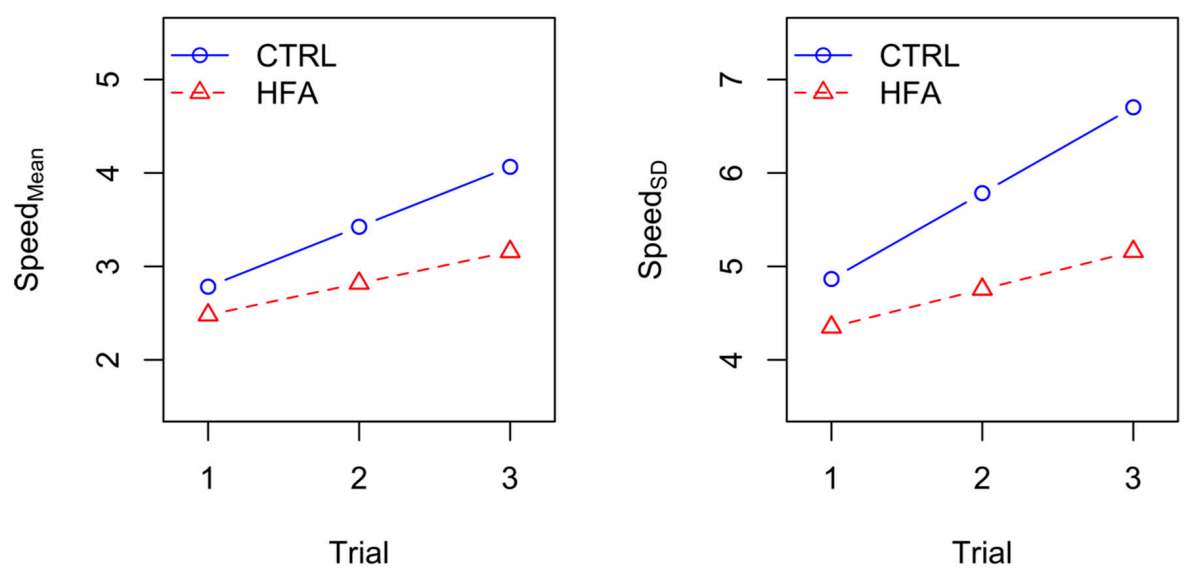

B

First Trial

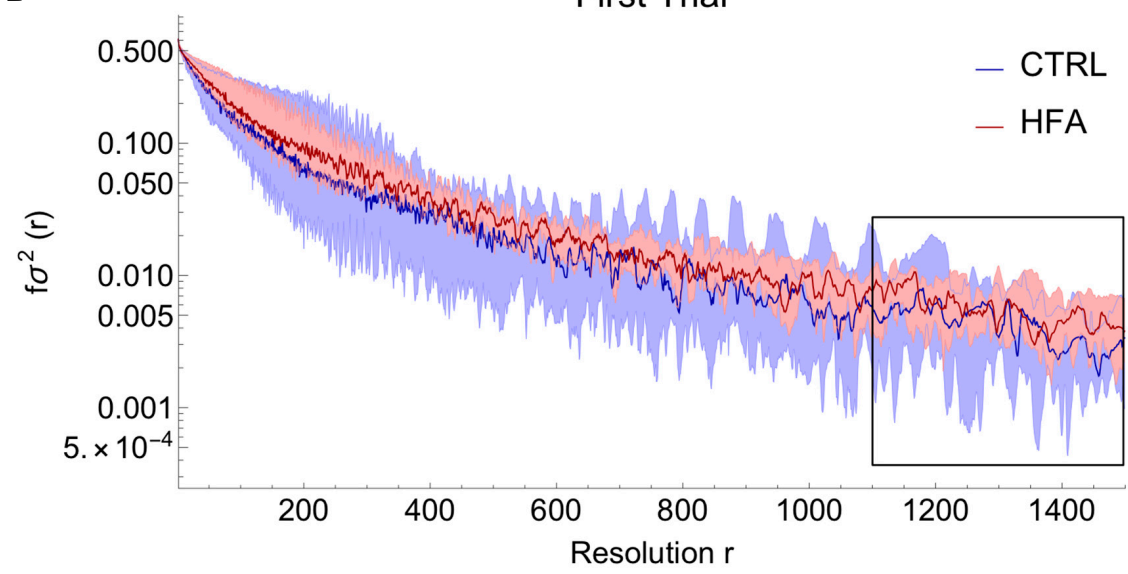

C

Third Trial

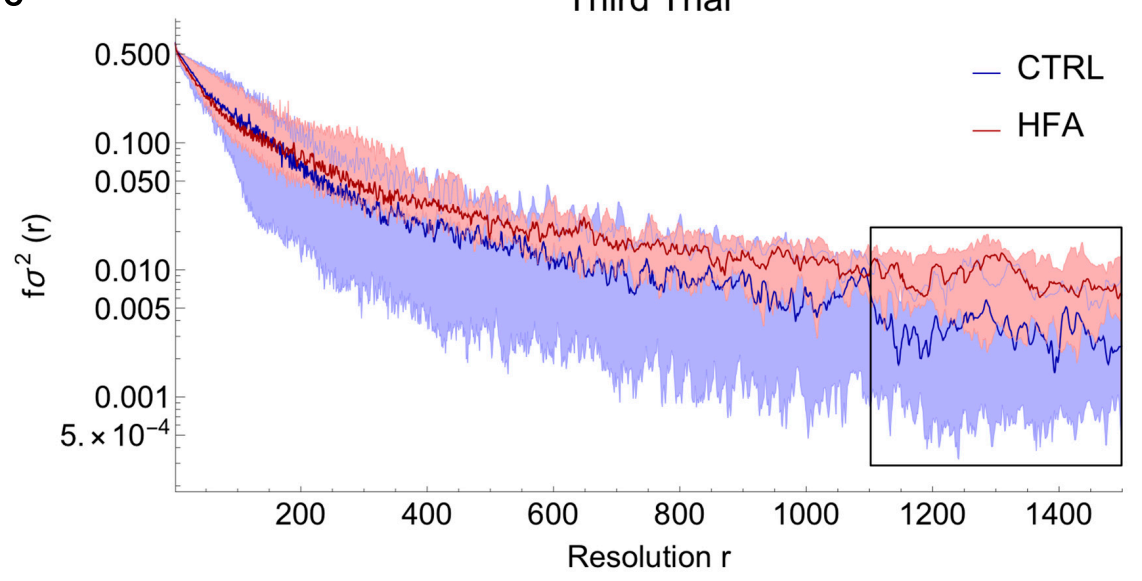

FIGURE 2 | Results. (A) Model fit for the mean of speed (absolute velocity) and the standard deviation of speed. (B,C) Coarse-graining analysis results: Median (dark curves) and region between 1st and 3rd quartiles (shaded areas) for controls (CTRL) and high-functioning autism individuals (HFA). Comparing the end (trial 3) with the start (trial 1) of the experiment, we find that HFA participants start to deviate from CTRL participants, especially for $r=1100$ to 1500 , i.e., an approximate temporal resolution of 22 to $30 \mathrm{~s}$. 


\section{Variability Pattern as Behavioral Marker in Patients With HFA}

The coarse-grained analysis was sensitive to the participants' different modes of movement understood as different variance values according to different time-scales. The fractional variance $\mathrm{f}^{2}{ }^{2}$ decreased with increasing resolution factors $r$, meaning that all participants made more frequently smaller jittery movements than slow ones with large periodicity.

Figures $2 \mathbf{B}, \mathbf{C}$ show that particularly in the third trial, the HFA group invests more variance in unidirectional and sustained movements (bigger $r$-values) when compared to the CTRL group. Notice that for the first trial the median values of both groups correspond, whereas for the third trial the median values deviate for coarser resolutions ( $r$ from 1100 to 1500, corresponding with an approximate temporal scale of 22 to $30 \mathrm{~s}$ ). The statistical analysis performed for each $r$ showed that from trial 1 to trial 3, HFA individuals progressively diverged from CTRL individuals (Supplementary Figure 4.1); further analysis of trialto-trial changes per group showed to be due to HFA individuals progressively investing more in large-scale movements and less in small-scale jittery movements (Supplementary Figure 4.2).

\section{DISCUSSION}

In this report, we presented a quantitative characterization of the movement patterns during a social contingency detection task, systematically comparing the movement of HFA and nonHFA participants who could interact with each other. Such "movements perspective" (Torres et al., 2013) has been used to develop behavioral markers, particularly when the social component is compromised, like in ASD (Torres et al., 2016; Whyatt and Torres, 2017b; Ryu and Torres, 2018). Our findings further support this perspective of taking motor aspects as proxies of social deficits.

Our results showed that an overall mutual coordination was reached at the dyad level, even in this highly constrained, minimal environment. This so-called complexity matching between participants during real-time interaction is consistent with our previous findings in non-autistic participants: $D_{\mathrm{a}, \mathrm{b}}$ Original $=12.47$, and $D_{\mathrm{a}, \mathrm{b}}$ Surrogate $=11.78$ (Zapata-Fonseca et al., 2016). Also, it goes in line with findings of embodied social competence during conversation in children with ASD (Romero et al., 2018). Additionally, our research supports the usage of computer-mediated and tactile interactions for understanding the relationship between movement-based coordination and social engagement in patients with ASD (Dautenhahn, 2007; Robins and Dautenhahn, 2014). We suggest that technology might fulfill a scaffolding role (Aresti-Bartolome and Garcia-Zapirain, 2014; Pennisi et al., 2016) at the level of movement coordination, but future research is required to disentangle the specific factors leading to such multi-scalar coordination.

Despite the mutual coordination, the individual's movement profiles showed differences between CTRL and AUT participants in relation to the trial number. Non-HFA participants increased their velocity of their movements much faster and in a more varied, if not "erratic" or unpredictable fashion across trials. The higher movement variability is consistent with the so-called healthy range of variability, not only in motor behaviors (Riley and Turvey, 2002; Romero et al., 2018), but also in other adaptive behaviors (Torres, 2013; Dotov et al., 2017; Dotov and Froese, 2018) and physiology dynamics (Rivera et al., 2016; Fossion et al., 2018). This suggests that non-HFA participants were less inhibited and more flexible in adapting their behavioral repertoire. Conversely, HFA participants consistently spent more time on large-scale movements and divested in smallscale jittery movements such as present during a mutual encounter, which indicates a preference for a more thorough, systematic and rational exploration of the virtual environment. Perhaps they focused on the social contingency detection task of clicking correctly, and hence less on engaging in social interaction for its own sake, whereas non-HFA persons appeared to "search for" and "enjoy" their partner, as has been anecdotally reported in other PCE studies (Auvray and Rohde, 2012). This interpretation is consistent with the reduced social motivation associated with autism, whereas the avoidance of jittery encounter movements and associated tactile stimulation may be related to ASD and hypersensitivity (Bottini, 2018; Clements et al., 2018).

\section{CONCLUSION}

In conclusion, our previous analysis showed that the HFA group was sensitive to the presence of the other, as shown by their clicking accuracy when identifying the other (ZapataFonseca et al., 2018). The present analysis found that the implicit movement coordination was also comparable with the complexity matching found in non-HFA pairs (Zapata-Fonseca et al., 2016). However, our results also suggest that HFA participants distinctively avoided situations of mutual perceptual crossing and instead preferred an objective perceptual strategy. This is consistent with gaze studies showing that HFA individuals tend to actively avoid mutual gaze during interaction and instead prefer to perceptually explore the rest of the other's body and environment (Guillon et al., 2014; Chevallier et al., 2015). Here, for the first time, we have revealed a similar pattern in the tactile modality.

Finally, our findings support previous research on movement profiles and their variability in ASD (Brincker and Torres, 2013; Torres and Denisova, 2016; Torres and Whyatt, 2017; Whyatt and Torres, 2017a, 2018), as well as on the value of the PCE and time-series analysis for quantitatively describing behaviors related to social cognition (Bedia et al., 2014; Zapata-Fonseca et al., 2016; Kojima et al., 2017). However, this work should be considered as preliminary because it is the first study to analyze the dynamic trajectories during this task in HFA, and motor and timing of movement deficits in ASD might be potential contributing factors to the effects found in this research and therefore require further studies. Importantly, the sample size was rather small and CTRL-CTRL pairs were not included here, so further analyses are required to clarify the contribution made by each individual to the interaction dynamics. 


\section{ETHICS STATEMENT}

This study was carried out in accordance with the recommendations of the guidelines of the Ethics Commission of the Medical Faculty of the University of Cologne (EthikKommission der Medizinischen Fakultät der Universität $\mathrm{zu}$ Köln) with written informed consent from all subjects. All subjects gave written informed consent in accordance with the Declaration of Helsinki. As the HFA volunteer participants were former patients of the Poliklinik für Psychiatrie und Psychotherapie, University Hospital of Cologne, their personal data are stored on the passwordprotected intranet (without access to the internet) of the University Hospital of Cologne. For screening purposes, test data from participants were retrieved, but were anonymised. The protocol was approved by the Ethics Commission of the Medical Faculty of the University of Cologne.

\section{AUTHOR CONTRIBUTIONS}

$\mathrm{BT}, \mathrm{KV}$, and LS conceived the study. BT designed and performed the experiments. LZ-F, BT, RF, and DD analyzed the data in close discussion with TF. LZ-F, DD, and BT wrote the first draft of the manuscript. All authors have made a substantial, direct and intellectual contribution to the work, and approved it for publication.

\section{REFERENCES}

Abney, D. H., Paxton, A., Dale, R., and Kello, C. T. (2014). Complexity matching in dyadic conversation. J. Exp. Psychol. Gen. 143, 2304-2315. doi: 10.1037/ xge0000021

Allan, D. W. (1966). Statistics of atomic frequency standards. Proc. IEEE 54, 221-230. doi: 10.1109/PROC.1966.4634

American Psychiatric Association and American Psychiatric Association DSM-5 Task Force. (2013). Diagnostic and Statistical Manual of Mental Disorders: DSM5, 5th edn. Washington, DC: American Psychiatric Association. doi: 10.1176/ appi.books. 9780890425596

Aresti-Bartolome, N., and Garcia-Zapirain, B. (2014). Technologies as support tools for persons with autistic spectrum disorder: a systematic review. Int. J. Environ. Res. Public Health 11, 7767-7802. doi: 10.3390/ijerph110807767

Auvray, M., and Rohde, M. (2012). Perceptual crossing: the simplest online paradigm. Front. Hum. Neurosci. 6:181. doi: 10.3389/fnhum.2012.00181

Bates, D., Mächler, M., Bolker, B., and Walker, S. (2015). Fitting linear mixed-effects models using lme4. J. Stat. Softw. 67, 1-48. doi: 10.18637/jss.v067.i01

Bedia, M. G., Aguilera, M., Gómez, T., Larrode, D. G., and Seron, F. (2014). Quantifying long-range correlations and 1/f patterns in a minimal experiment of social interaction. Front. Psychol. 5:1281. doi: 10.3389/fpsyg.2014.01281

Bottini, S. (2018). Social reward processing in individuals with autism spectrum disorder: a systematic review of the social motivation hypothesis. Res. Autism Spectr. Disord. 45, 9-26. doi: 10.1016/j.rasd.2017.10.001

Brincker, M., and Torres, E. B. (2013). Noise from the periphery in autism. Front. Integr. Neurosci. 7:34. doi: 10.3389/fnint.2013.00034

Chevallier, C., Parish-Morris, J., McVey, A., Rump, K. M., Sasson, N. J., Herrington, J. D., et al. (2015). Measuring social attention and motivation in autism spectrum disorder using eye-tracking: stimulus type matters. Autism Res. 8, 620-628. doi: 10.1002/aur.1479

Clements, C. C., Zoltowski, A. R., Yankowitz, L. D., Yerys, B. E., Schultz, R. T., and Herrington, J. D. (2018). Evaluation of the social motivation hypothesis of

\section{FUNDING}

We acknowledge financial support from DGAPA-PAPIIT projects of the Universidad Nacional Autónoma de México: IA105017 (RF and LZ-F) and IA104717 (TF), Consejo Nacional de Ciencia y Tecnología (CONACyT) projects 167441 (RF and LZ-F), the scholarship 638215 to LZ-F granted by the CONACyT, the Newton Advanced Fellowship awarded to RF by the Academy of Medical Sciences, through the UK Government's Newton, and the Marie Curie Intra-European Fellowship "SOCIAL BRAIN" awarded to BT.

\section{ACKNOWLEDGMENTS}

We thank Charles Lenay and Dominique Aubert from the Université de Technologie de Compiègne for making the TACTOS hardware and software available to the University Hospital Cologne, and for providing technical support. LZ-F would like to specially thank Jesús Naveja and Lilia Fonseca for interesting discussions.

\section{SUPPLEMENTARY MATERIAL}

The Supplementary Material for this article can be found online at: https://www.frontiersin.org/articles/10.3389/fpsyg. 2018.02760/full\#supplementary-material

autism: a systematic review and meta-analysis. JAMA Psychiatry 75, 797-808. doi: 10.1001/jamapsychiatry.2018.1100

Cook, J. L., Blakemore, S.-J., and Press, C. (2013). Atypical basic movement kinematics in autism spectrum conditions. Brain 136, 2816-2824. doi: 10.1093/ brain/awt 208

Dautenhahn, K. (2007). Socially intelligent robots: dimensions of human-robot interaction. Philos. Trans. R. Soc. Lond. B Biol. Sci. 362, 679-704. doi: 10.1098/ rstb.2006.2004

De Jaegher, H. (2013). Embodiment and sense-making in autism. Front. Integr. Neurosci. 7:15. doi: 10.3389/fnint.2013.00015

Dotov, D., and Froese, T. (2018). Entraining chaotic dynamics: a novel movement sonification paradigm could promote generalization. Hum. Mov. Sci. 61, 27-41. doi: 10.1016/j.humov.2018.06.016

Dotov, D. G., Bayard, S., Cochen de Cock, V., Geny, C., Driss, V., Garrigue, G., et al. (2017). Biologically-variable rhythmic auditory cues are superior to isochronous cues in fostering natural gait variability in Parkinson's disease. Gait Posture 51, 64-69. doi: 10.1016/j.gaitpost.2016.09.020

Fossion, R., Rivera, A. L., and Estañol, B. (2018). A physicist's view of homeostasis: how time series of continuous monitoring reflect the function of physiological variables in regulatory mechanisms. Physiol. Meas. 39:84007. doi: 10.1088/1361-6579/ aad8db

Fossion, R., Rivera, A. L., Toledo-Roy, J. C., Ellis, J., and Angelova, M. (2017). Multiscale adaptive analysis of circadian rhythms and intradaily variability: application to actigraphy time series in acute insomnia subjects. PLoS One 12:e0181762. doi: 10.1371/journal.pone.0181762

Froese, T. (2018). "Searching for the conditions of genuine intersubjectivity: from agent-based models to perceptual crossing experiments," in The Oxford Handbook of $4 E$ Cognition, eds L. D. B. A. Newen and S. Gallagher (Oxford: Oxford University Press).

Froese, T., and Di Paolo, E. A. (2011). The enactive approach: theoretical sketches from cell to society. Pragmat. Cogn. 19, 1-36. doi: 10.1075/pc.19.1.01fro 
Froese, T., Iizuka, H., and Ikegami, T. (2014). Embodied social interaction constitutes social cognition in pairs of humans: a minimalist virtual reality experiment. Sci. Rep. 4:3672. doi: 10.1038/srep03672

Froese, T., Stanghellini, G., and Bertelli, M. O. (2013). Is it normal to be a principal mindreader? revising theories of social cognition on the basis of schizophrenia and high functioning autism-spectrum disorders. Res. Dev. Disabil. 34, 1376-1387. doi: 10.1016/j.ridd.2013.01.005

Froese, T., and Zapata-Fonseca, L. (2017). Commentary: alignment in social interactions. Front. Psychol. 8:1249. doi: 10.3389/fpsyg.2017.01249

Fuchs, T. (2015). Pathologies of intersubjectivity in autism and schizophrenia. J. Conscious. Stud. 22, 191-214.

Gallagher, S. (2004). Understanding interpersonal problems in autism: interaction theory as an alternative to theory of mind. Philos. Psychiatr. Psychol. 11, 199-217. doi: 10.1353/ppp.2004.0063

Guillon, Q., Hadjikhani, N., Baduel, S., and Rogé, B. (2014). Visual social attention in autism spectrum disorder: insights from eye tracking studies. Neurosci. Biobehav. Rev. 42, 279-297. doi: 10.1016/j.neubiorev.2014.03.013

Kojima, H., Froese, T., Oka, M., Iizuka, H., and Ikegami, T. (2017). A sensorimotor signature of the transition to conscious social perception: co-regulation of active and passive touch. Front. Psychol. 8:1778. doi: 10.3389/fpsyg.2017.01778

Kunde, W., Weller, L., and Pfister, R. (2018). Sociomotor action control. Psychon. Bull. Rev. 25, 917-931. doi: 10.3758/s13423-017-1316-6

Lenay, C. (2008). Médiations techniques des interactions perceptives: rencontres tactiles dans les environnements numériques partagés. Soc. Sci. Inf. 47, 331-352. doi: $10.1177 / 0539018408092576$

Pennisi, P., Tonacci, A., Tartarisco, G., Billeci, L., Ruta, L., Gangemi, S., et al. (2016). Autism and social robotics: a systematic review. Autism Res. 9, 165-183. doi: $10.1002 /$ aur. 1527

Riley, M. A., and Turvey, M. T. (2002). Variability of determinism in motor behavior. J. Mot. Behav. 34, 99-125. doi: 10.1080/00222890209601934

Rivera, A. L., Estañol, B., Sentíes-Madrid, H., Fossion, R., Toledo-Roy, J. C., Mendoza-Temis, J., et al. (2016). Heart rate and systolic blood pressure variability in the time domain in patients with recent and long-standing diabetes mellitus. PLoS One 11:e0148378. doi: 10.1371/journal.pone.0148378

Robins, B., and Dautenhahn, K. (2014). Tactile interactions with a humanoid robot: novel play scenario implementations with children with autism. Int. J. Soc. Robot. 6, 397-415. doi: 10.1007/s12369-014-0228-0

Romero, V., Fitzpatrick, P., Roulier, S., Duncan, A., Richardson, M. J., and Schmidt, R. C. (2018). Evidence of embodied social competence during conversation in high functioning children with autism spectrum disorder. PLoS One 13:e0193906. doi: 10.1371/journal.pone.0193906

Ryu, J., and Torres, E. B. (2018). Characterization of sensory-motor behavior under cognitive load using a new statistical platform for studies of embodied cognition. Front. Hum. Neurosci. 12:116. doi: 10.3389/fnhum.2018.00116

Schilbach, L. (2016). Towards a second-person neuropsychiatry. Philos. Trans. $R$. Soc. Lond. B Biol. Sci. 371:20150081. doi: 10.1098/rstb.2015.0081

Schilbach, L., Timmermans, B., Reddy, V., Costall, A., Bente, G., Schlicht, T., et al. (2013). Toward a second-person neuroscience. Behav. Brain Sci. 36, 393-414. doi: $10.1017 /$ S0140525X12000660

Singer, J. D., and Willett, J. B. (2003). Applied Longitudinal Data Analysis. Oxford: Oxford University Press. doi: 10.1093/acprof:oso/9780195152968.001.0001

Słowiński, P., Zhai, C., Alderisio, F., Salesse, R., Gueugnon, M., Marin, L., et al. (2016). Dynamic similarity promotes interpersonal coordination in joint action. J. R. Soc. Interface 13:20151093. doi: 10.1098/rsif.2015.1093
Torres, E., and Whyatt, C. (eds). (2017). Autism: The Movement Sensing Perspective. Boca Raton, FL: CRC Press, doi: 10.1201/9781315372518

Torres, E. B. (2013). Atypical signatures of motor variability found in an individual with ASD. Neurocase 19, 150-165. doi: 10.1080/13554794.2011.654224

Torres, E. B., Brincker, M., Isenhower, R. W., Yanovich, P., Stigler, K. A., Nurnberger, J. I., et al. (2013). Autism: the micro-movement perspective. Front. Integr. Neurosci. 7:32. doi: 10.3389/fnint.2013. 00032

Torres, E. B., and Denisova, K. (2016). Motor noise is rich signal in autism research and pharmacological treatments. Sci. Rep. 6:37422. doi: 10.1038/srep37422

Torres, E. B., Isenhower, R. W., Nguyen, J., Whyatt, C., Nurnberger, J. I., Jose, J. V., et al. (2016). Toward precision psychiatry: statistical platform for the personalized characterization of natural behaviors. Front. Neurol. 7:8. doi: $10.3389 /$ fneur.2016.00008

Torres, E. B., Mistry, S., Caballero, C., and Whyatt, C. P. (2017). Stochastic signatures of involuntary head micro-movements can be used to classify females of ABIDE into different subtypes of neurodevelopmental disorders. Front. Integr. Neurosci. 11:10. doi: 10.3389/fnint.2017. 00010

West, B. J., Geneston, E. L., and Grigolini, P. (2008). Maximizing information exchange between complex networks. Phys. Rep. 468, 1-99. doi: 10.1016/j. physrep.2008.06.003

Whyatt, C. P., and Torres, E. B. (2017a). "ADOS: the physiology approach to assess social skills and communication in autism spectrum disorder," in Autism: The Movement Sensing Perspective Frontiers in Neuroscience, eds E. Torres and C. Whyatt (Boca Raton, FL: CRC Press), 103-118. doi: 10.1201/978131537 2518-11

Whyatt, C. P., and Torres, E. B. (2017b). "The social-dance: decomposing naturalistic dyadic interaction dynamics to the "micro-level," in Proceedings of the 4th International Conference on Movement Computing - MOCO '17, ed. K. Niehaus (New York, NY: ACM Press), 1-8. doi: 10.1145/3077981.3078055

Whyatt, C. P., and Torres, E. B. (2018). Autism research: an objective quantitative review of progress and focus between 1994 and 2015. Front. Psychol. 9:152. doi: 10.3389/fpsyg.2018.01526

Zapata-Fonseca, L., Dotov, D., Fossion, R., and Froese, T. (2016). Timeseries analysis of embodied interaction: movement variability and complexity matching as dyadic properties. Front. Psychol. 7:1940. doi: 10.3389/fpsyg.2016. 01940

Zapata-Fonseca, L., Froese, T., Schilbach, L., Vogeley, K., and Timmermans, B. (2018). Sensitivity to social contingency in adults with high-functioning autism during computer-mediated embodied interaction. Behav. Sci. 8:E22. doi: $10.3390 / \mathrm{bs} 8020022$

Conflict of Interest Statement: The authors declare that the research was conducted in the absence of any commercial or financial relationships that could be construed as a potential conflict of interest.

Copyright (c) 2019 Zapata-Fonseca, Dotov, Fossion, Froese, Schilbach, Vogeley and Timmermans. This is an open-access article distributed under the terms of the Creative Commons Attribution License (CC BY). The use, distribution or reproduction in other forums is permitted, provided the original author(s) and the copyright owner(s) are credited and that the original publication in this journal is cited, in accordance with accepted academic practice. No use, distribution or reproduction is permitted which does not comply with these terms. 